Fig. 1. Opportunities for antimicrobial stewardship programs to assist COVID-19 response preparation and planning efforts.

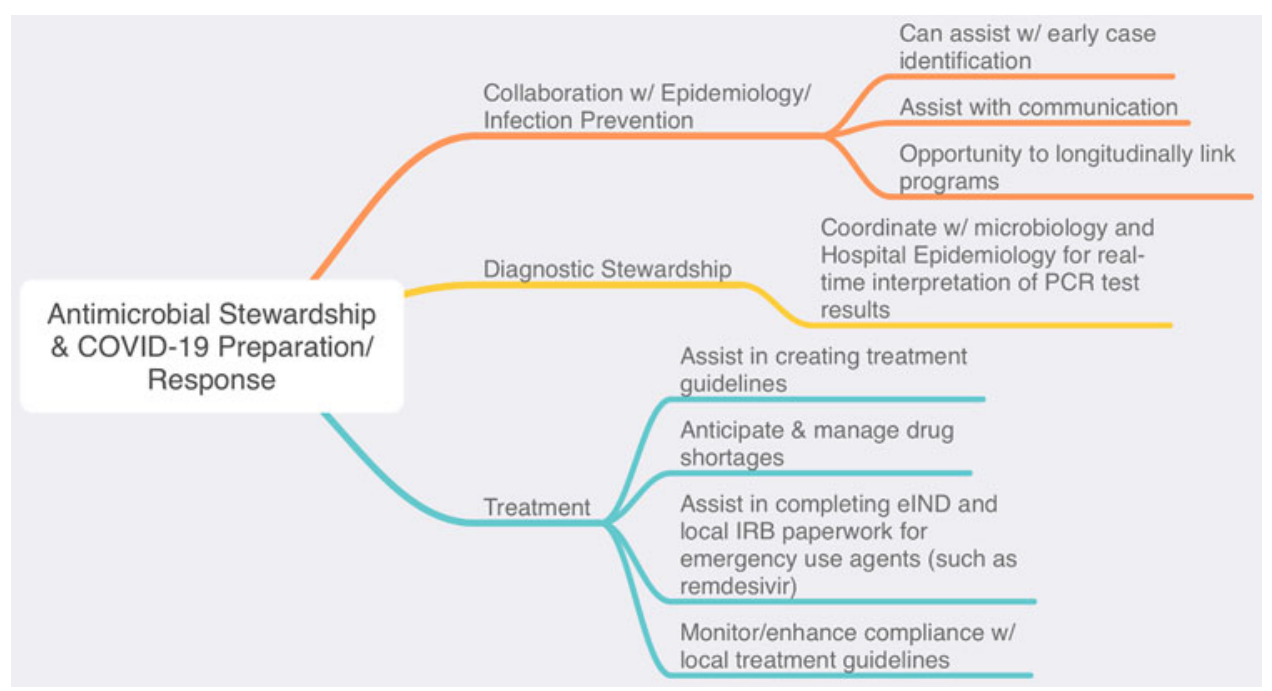

Conflicts of interest. All authors report no conflicts of interest relevant to this article.

\section{References}

1. Banach DB, Johnston BL, Al-Zubeidi D, et al. Outbreak response and incident management: SHEA guidance and resources for healthcare epidemiologists in United States acute-care hospitals. Infect Control Hosp Epidemiol 2017;38:1393-1419.

2. Abbas S, Stevens MP. The role of the hospital epidemiologist in antibiotic stewardship. Med Clin North Am 2018;102:873-882.

3. Doernberg SB, Abbo LM, Burdette SD, et al. Essential resources and strategies for antibiotic stewardship programs in the acute care setting. Clin Infect Dis 2018;67:1168-1174.
4. Young BE, Ong SWX, Kalimuddin S, et al. Epidemiologic features and clinical course of patients infected with SARS-CoV-2 in Singapore. JAMA 2020. doi:10.1001/jama.2020.3204

5. Coronavirus (COVID-19) Supply Chain Update. US Food and Drug Administration website. https://www.fda.gov/news-events/press-announcements/ coronavirus-covid-19-supply-chain-update. Accessed March 4, 2020.

6. Approved: new antimicrobial stewardship standard. The Joint Commission website. https://www.jointcommission.org/-/media/enterprise/tjc/importedresource-assets/documents/new_antimicrobial_stewardship_standardpdf. pdf?db=web\&hash=69307456CCE435B134854392C7FA7D76. Published July 2015. Accessed March 5, 2020.

\title{
Protecting Chinese healthcare workers while combating the 2019 novel coronavirus
}

\author{
Pengcheng Zhou $M D^{1}$, Zebing Huang $\mathrm{MD}^{2}$, Yinzong Xiao $\mathrm{MD}^{2,3}$, Xun Huang $\mathrm{MD}^{1}$ and Xue-Gong Fan $\mathrm{MD}^{2}$ \\ ${ }^{1}$ Infection Control Centre, Xiangya Hospital, Central South University, Changsha, China, ${ }^{2}$ Hunan Key Laboratory of Viral Hepatitis \& Department of Infectious \\ Diseases, Xiangya Hospital, Central South University, Changsha, China and ${ }^{3}$ Burnet Institute, St Vincent's Hospital Melbourne, and University of Melbourne, \\ Melbourne, Australia
}

To the Editor-Hospital-associated transmission is an important route of spreading the 2019 novel coronavirus SARS-CoV-2 and pneumonia (coronavirus disease 2019, COVID-19). ${ }^{1}$ Healthcare workers (HCWs) are at high risk while combating COVID-19 at the very front line, and nosocomial outbreaks among HCWs are not unusual in similar settings. The 2003 severe acute respiratory syndrome (SARS) outbreak led to $>966 \mathrm{HCW}$ infections with

Author for correspondence: Xue-Gong Fan, E-mail: xgfan@hotmail.com Or Xun Huang, E-mail: huangxun224@126.com

Cite this article: Zhou P, et al. (2020). Protecting Chinese healthcare workers while combating the 2019 novel coronavirus. Infection Control \& Hospital Epidemiology, 41: 745-746, https://doi.org/10.1017/ice.2020.60
$1.4 \%$ deaths in mainland China. ${ }^{2}$ As of February 11, 2020, 3,019 HCWs might have been infected with SARS-CoV-2 in China, and 1,716 HCW cases of COVID-19 have been confirmed by nucleic acid testing ${ }^{3}$ At least $6 \mathrm{HCW}$ s have died, including the famous whistleblower Dr Li Wenliang. In view of this severe situation, we are recommending urgent interventions to help to protect HCWs.

A few aspects of COVID-19 have created a more severe situation than expected among HCWs. First, many infected individuals present with a typical symptoms, such as gastrointestinal symptoms and fatigue, or are asymptomatic. ${ }^{4}$ This situation may lead to a lack of recognition of the infection while patients are highly 
contagious. Furthermore, HCWs have not been not well prepared for this sudden COVID-19 outbreak, especially in departments other than infectious diseases. In Wuhan at the beginning of the outbreak, there was a general lack of awareness among HCWs to take precautions, and inadequate training among HCWs was noted, with staff incorrectly wearing personal protective equipment (PPE). In fact, $\sim 30$ HCWs in the Wuhan Mental Health Hospital were reported to be infected. ${ }^{5}$ Third, no point-of-care diagnostic assay was available in hospitals before late January 2019. In addition, the positive rate of the SARS-CoV-2 nucleic acid test kit remains relatively low even at present, and many patients have been diagnosed after $>4$ tests. These factors led to a diagnostic delay and opportunities for exposure among HCWs. Fourth, a good many tertiary and secondary hospitals are experiencing shortages of PPE and are calling for donations. HCWs have to use daily plastic products (photographic film, plastic wrap, file bag, and so forth) to make simple PPE. Lastly, some COVID-19 patients were admitted to the other departments by concealing their epidemiological history, which led to unnecessary exposure of HCWs.

Much can be done! We hope all countries and all people in the world can support the brave men and women on the front line of combating SARS-CoV-2. More PPE should be produced or imported, and it should be delivered to hospitals quickly. Training of HCWs to identify suspicious cases and to use PPE properly is urgently needed, especially for HCWs in departments other than infectious diseases. Furthermore, concealing medical history should have legal consequences.

\section{Acknowledgments. None.}

Financial support. No financial support was provided relevant to this article.

Conflicts of interest. All authors report no conflicts of interest relevant to this article.

\section{References}

1. Chan JF, Yuan S, Kok KH, et al. A familial cluster of pneumonia associated with the 2019 novel coronavirus indicating person-to-person transmission: a study of a family cluster. Lancet 2020;395:514-523.

2. General epidemic situation of SARS in China (May 29, 2003). National Health Commission of the People's Republic of China website. http:// www.nhc.gov.cn/wjw/zcjd/201304/2e198946322b4b9ab3972565ff3db8c6. shtml. Published 2003. Accessed February 20, 2020.

3. The Novel Coronavirus Pneumonia Emergency Response Epidemiology Team, Chinese Center for Disease Control and Prevention. The epidemiological characteristics of an outbreak of 2019 novel coronavirus diseases (COVID-19) in China. Chin J Epidemiol 2020;42:145-151.

4. Diagnosis and treatment program of novel coronavirus pneumonia, 5th edition. National Health Commission of the People's Republic of China website. http:// www.nhc.gov.cn/yzygj/s7653p/202002/d4b895337e19445f8d728fcafle3e13a. shtml. Published 2020. Accessed February 20, 2020.

5. Nosocomial infection outbreak in Wuhan mental health center, and about 80 healthcare workers and patients were infected with novel coronavirus pneumonia. Peoples Daily website. http://society.people.com.cn/n1/2020/ 0209/c1008-31577664.html. Published February 8, 2020. Accessed February $20,2020$.

\title{
The role of masks and respirator protection against SARS-CoV-2
}

\author{
Qiang Wang MD, $\mathrm{PhD}^{1,2}$ and Chaoran Yu MD, $\mathrm{PhD}^{1,2}$ \\ ${ }^{1}$ Fudan University Shanghai Cancer Center, Fudan University, Shanghai, 200025, PR China and ${ }^{2}$ Department of Oncology, Shanghai Medical College, Fudan \\ University, Shanghai, 200025, PR China
}

To the Editor - The outbreak of COVID-19, the novel coronavirus SARS-CoV-2 infection, was first reported on December 31, 2019, in Wuhan, a central city in China. The SARS-CoV-2 virus has infected $>30,000$ people in a very short time, with hundreds of deaths. ${ }^{1}$ COVID-19 continues to be a flaming infectious disease across the world. However, many details of the biological features of this virus remain largely unknown.

SARS-CoV-2 is the third coronavirus to have threatened global public health in the past 20 years, following severe acute respiratory syndrome coronavirus (SARS-CoV) in 2002 and Middle East respiratory syndrome coronavirus (MERS-CoV) in $2012 .^{2}$ In an updated COVID-19 report, Wang et $\mathrm{al}^{3}$ indicated that the median age of death was 75 years, and fever (64.7\% of deaths) and cough (52.9\% of deaths) were identified as initial clinical manifestation. ${ }^{3}$ Genomic characterization of samples from 9 COVID-19 patients indicated that SARS-CoV-2 had $88 \%$ identity with 2 bat-derived SARS-like coronaviruses (bat-SL-CoVZC45 and bat-SL-CoVXC21),

Author for correspondence: Chaoran Yu, E-mail: chaoran_yu@yeah.net or chaoran_yu@ sjtu.edu.cn

Cite this article: Wang Q and Yu C. (2020). The role of masks and respirator protection against SARS-CoV-2 . Infection Control \& Hospital Epidemiology, 41: 746-747, https:// doi.org/10.1017/ice.2020.83 whereas these bat viruses had 79\% identity with SARS-CoV and 50\% identity with MERS-CoV. ${ }^{4}$ A phylogenetic analysis has indicated that SARS-CoV-2 belongs to the genus Betacoronavirus and the subgenus sarbecovirus. ${ }^{4}$

Given that large-scale spread of this virus is now occurring around the world, the identification of cases and the containment of possible routes of spread have become a priority. Increasing risk has narrowed the window of opportunity for effective abatement of COVID-19. Containing an outbreak becomes much more complicated and challenging when hospitalized populations are exposed. Notably, Zhou et $\mathrm{al}^{5}$ recommended urgent interventions for the protection of Chinese healthcare workers against SARS-CoV-2. In fact, this warning raised attention regarding the role of daily-use N95 respirators and masks during this pandemic.

Given the similarity of SARS-CoV-2 and SARS-CoV, initial political recommendations in China highlighted the use of masks and N95 respirators for protection against SARS-CoV-2. Wearing masks and respirators and self-isolation at home has been issued as a practice guideline for public in China. Of 213 medical staff with no mask, 10 were infected by SARS-CoV-2, but none of the 278 medical staff wearing N95 respirators was infected. ${ }^{6}$ Interestingly, a higher risk of infection has been noted in male 